\title{
DESTRUCTION DES ALGUES ENVAHISSANTES DANS LES ETANGS
}

\author{
par Jean MARTIN et Marcel LEFEVRE \\ Directeur du Centre de Recherches Hydrobiologiques du C. N. R. S.
}

Le rôle des Algues dans les collections d'eau naturelles est primordial, mais, suivant leur nature, elles peuvent avoir une action hautement favorisante ou au contraire fort nuisible.

Lorsqu'elles sont unicellulaires, très petites et à membrane mince, elles constituent une nourriture de choix pour le Zooplancton qui se développe alors en grande abondance, procurant ainsi à l'étang un rendement piscicole très élevé.

Lorsqu'elles sont filamenteuses et qu'elles se développent en grande masse, elles présentent au contraire une foule d'inconvénients. Étant trop volumineuses pour pouvoir servir de nourriture aux éléments du zooplancton, elles épuisent en pure perte les sels minéraux de l'étang. Elles entravent la circulation des Poissons, des petits animaux qui leur servent de nourriture et constituent des pièges à alevins fort peu recommandables.

Lorsqu'elles finissent par former " fleur d'eau ", elles peuvent couvrir l'étang d'un tapis continu, parfois épais de plusieurs centimètres, qui constitue un écran à peu près complètement opaque à la lumière. De ce fait, et en raison aussi de substances métaboliques antagonistes qu'elles secrètent, aucune Algue unicellulaire utile ne peut se développer dans la masse d'eau qu'elles recouvrent. Le zooplancton est alors très pauvre dans l'étang et le rendement piscicole s'en trouve gravement affecté.

Enfin, si pour une raison quelconque la fleur d'eau vient à se décomposer brusquement, tout l'oxygène dissous dans l'eau se trouve absorbé par la fermentation. Les Poissons périssent alors soit asphyxiés, en raison du manque d'oxygène, soit empoisonnés par les substances toxiques secrétées par les Bactéries responsables de la fermentation. 
Dernièrement, un étang de pisciculture d'un hectare et demi environ, l'étang des Joncs, situé sur la propriété du "Ciran " (Loiret) se trouvait justement recouvert d'une fleur d'eau à Spirogyra si importante qu'il ne restait pas même quelques mètres carrés d'eau libre et que la circulation en barque $\mathrm{y}$ était devenue fort pénible, sinon impossible. Cette fleur d'eau qui existait déjà l'année précédente, avait résisté à un court assec et à l'hiver.

On tenta donc de s'en débarrasser par le sulfate de cuivre et voici dans quelles conditions :

Un essai préliminaire fut d'abord effectué.

Un bac de tôle goudronnée (le goudronnage ayant été fait depuis très longtemps ne pouvait intervenir dans l'expérience) fut amené au bord de l'étang.

Deux cents litres de l'eau de l'étang contenant une forte proportion de Spirogyres furent introduits dans le bac. D'autre part, on fit dissoudre 1 gramme de sulfate de cuivre dans un peu d'eau et le liquide obtenu fut mélangé intimement à l'eau du bac.

La proportion de sulfate de cuivre dans le bac était donc de 1/200.000 environ.

Au bout de huit jours, il n'y avait plus trace d'Algues à la surface et dans la masse mème de l'eau qui était devenue claire. Des Entomostracés d'origine y vivaient normalement et n'avaient pas été touchés par le sulfate de cuivre. Devant ce résultat encourageant, il fut décidé de traiter l'étang par le même procédé, avec cependant quelques variantes.

Tout d'abord, l'effet du sulfate de cuivre à $1 / 200.000$ nous ayant paru particulièrement rapide, nous avons pensé que le $1 / 500.000$ pourrait peut-être se montrer suffisamment efficace, d'autant plus que des conditions spéciales allaient permettre un mode particulier d'application.

En effet, l'épaisseur de la fleur d'eau était telle, qu'elle nous semblait pouvoir supporter sans s'enfoncer des cristaux de sulfate de cuivre de la grosseur d'un grain de blé. Il devait donc être possible de semer le sulfate de cuivre à sec, à la volée, sur la fleur d'eau, au moyen d'une barque. Ces cristaux se dissolvant lentement au contact des Algues, baigneraient celles-ci dans une solution momentanément très concentrée et particulièrement efficace, tandis que la teneur finale de l'eau de l'étang en sulfate de cuivre, après destruction de la fleur d'eau, ne dépasserait pas les limites compatibles avec la vie animale.

D'après nos calculs 50 kilos de sulfate de cuivre devaient être suffisants pour traiter l'étang au 1/500.000, mais, finalement, il fut décidé d'opérer en deux fois.

Une première application de 25 kilos de sulfate de cuivre fut donc faite au début de Juin. Le 12 Juin on pouvait déjà effectuer les constatations suivantes :

Présence de plusieurs zones entièrement libérées d'Algues, zones de plusieurs dizaines de mètres carrés chacune. 
Changement de couleur de la fleur d'eau qui, de vert jaunâtre qu'elle était, est passée au gris mauve.

La fleur d'eau est devenue molle. Elle est en voie de décomposition et ne peut plus s'opposer au passage de la barque.

Sous la fleur d'eau, et dans les espaces déjà entièrement libérés, présence d'une matière mucilagineuse noire formée d'Algues déjà presque complètement décomposées. Cette substance se dépose sur le fond de l'étang et sur les objets immergés.

Présence d'éléments du zooplancton bien vivants.

En résumé, l'opération était en bonne voie de réussite, la fleur d'eau morte et en décomposition ne se maintenant encore partiellement en surface que grâce aux bulles de gaz provenant de la fermentation.

Le 20 Juin au matin, toute la partie centrale de l'étang était entièrement dégagée, mais la partie marginale était encore encombrée.

Le 20 au soir, un violent orage accompagné de grêle sévit sur la région.

Le 21 au matin, il n'y a plus trace de fleur d'eau à la surface de l'étang : le brassage de l'eau avait dégagé les bulles de gaz du magma en décomposition et celui-ci était tombé au fond.

L'eau était très claire, un peu brune et présentait encore un zooplancton bien vivant.

Mais les Poissons avaient-ils résisté au traitement? La fermentation des Algues ne les avait-elle pas tués?

Des nasses tendues dans l'étang au début de Juillet nous rassurèrent sur ce point. Les brochets eux-mêmes avaient résisté. La concentration du sulfate de cuivre dans l'eau ne devait d'ailleurs pas, après cette première application, dépasser $1 / 1.000 .000$.

Dans les premiers jours d'Août, quelques formations isolées de Spirogyres étant de nouveau apparues, une seconde application de $\mathrm{SO}_{4} \mathrm{CU}$ au 1/1.000.000 fut décidée.

Les Algues furent rapidement détruites et ne se manifestèrent plus, ni cette même année, ni l'année suivante.

Encouragés par ce succès, nous avons poursuivi nos essais dans des bassins de garde de pisciculture.

Ces bassins, alimentés par un faible courant d'eau, sont souvent encombrés d'une végétation gênante d'Algues filamenteuses.

Un bassin de 130 mètres cubes fut traité de la façon suivante :

500 grammes de cristaux de sulfate de cuivre furent enfermés dans un sac de toile grossière et le sac, maintenu dans le filet d'eau d'arrivée.

Le sulfate de cuivre fut lentement dissous et diffusa dans le bassin.

La concentration fut donc, au début, voisine de 1/250.000 et s'affaiblit petit à petit puisque l'eau était lentement renouvelée.

Toutes les Algues envahissantes furent détruites en quelques jours et ne reparurent pas de la saison.

Ces essais montrent bien que la destruction des Algues par le sulfate de cuivre est un procédé efficace, d'application simple et assez peu onéreux. 
Cependant. les doses à employer ne peuvent être fixées à l'avance avec précision. Elles dépendent, en effet, de la minéralisation de l'eau traitée.

Nos essais ont été effectués en Sologne, région où les eaux sont très peu minéralisées, très pauvres en calcium.

Dans des eaux riches, à extrait sec élevé, on observe souvent une précipitation du cuivre sous forme de composés très peu solubles, donc beaucoup moins actifs. Il y a lieu, dans ce cas, de forcer la dose. Des essais préliminaires effectués dans des bocaux pourront rendre les plus grands services dans l'appréciation des quantités de $\mathrm{So}_{4} \mathrm{Cu}$ à employer. 